\title{
Structure-activity relationship of HER2 receptor targeting peptide and its derivatives in targeted tumor therapy
}

\author{
Afrodité Emese Adorján ${ }^{1}$, Szilvia Bósze ${ }^{2}$, Ildikó Szabó ${ }^{2}$, Gábor Mezó ${ }^{1}$ \\ ${ }^{1}$ Eötvös Loránd University, Hungary \\ ${ }^{2}$ MTA-ELTE Research Group of Peptide Chemistry, Hungary
}

https://doi.org/10.17952/35EPS.2018.320

\section{Introduction}

In normal cells, epidermal growth factor receptor 2 (HER2/ErbB2) plays a vital role in various cellular processes and the expression level of HER2 remains stable. When overexpression of HER2 occurs, it can disrupt the dynamic balance of many cellular processes and lead to uncontrolled tumor growth [1].

Breast cancer is one of the leading causes of death worldwide. In $20 \%$ of all cases, HER2 is found on breast cancer tissues. The overexpression of HER2 leads to an aggressive course of disease. Recently, targeted tumor therapy became a promising research area based on the increased selectivity of antitumor drugs attached to a targeting molecule, which binds to tumor specific antigens/receptors. Since HER2 might be a good target to prevent tumor growth, current researches are directed to discover HER2 targeting moieties for drug delivery. Trastuzumab-MCC-DM1 (Kadcyla ${ }^{\circledR}$ ), an antibody-drug conjugate (ADC) is on the market now [2]. In spite of the benefits of ADCs (e.g. long half-life in circulation, high selectivity), they also have some disadvantages (e.g. low tissue penetration, high costs). Therefore, small molecule-drug conjugates (SMDCs) based on peptides as targeting moieties might have advantages over ADCs [3]. Appropriate homing peptides can be selected by phage display or molecular dynamic (MD) simulations. In our research, KCCYSL peptide selected by phage display technique [4], as well as GYYNPT peptide that binds selectively to HER2 according to MD modelling [5] were chosen. The in vitro cellular uptake profile of the peptides and their derivatives was studied by HER2+ cancer cells.

\section{Results}

The N-terminally 5(6)-carboxyfluorescein (CF) labelled KCCYSL-NH 2 peptide and its derivatives (one or both of the cysteines were replaced by Ser or Ala) were synthesized by SPPS using Fmoc/tBu strategy. Furthermore, CF-GYYNPT- $\mathrm{NH}_{2}$ was prepared (Table 1.) for cellular uptake studies. The in vitro cellular uptake was measured by flow cytometry on MDA-MB-453 HER2+ cancer cells (medium expression level). The results indicate that CF-KCCYSL-NH ${ }_{2}$ and its modified analogs were only moderately taken up by the cells, while CF-GYYNPT$\mathrm{NH}_{2}$ did not internalize. The internalization profile of KCCYSL was not significantly influenced by the amino acid replacements in the peptide sequence (Figure 1A).

Table 1: Characteristics of the CF-labelled peptides developed for HER2 receptor recognition

\begin{tabular}{|c|c|c|c|}
\hline Compounds & $\operatorname{HPLC}\left(R_{t} / \mathrm{min}\right)$ & $\mathrm{MW}_{\text {calc }}[\mathrm{M}+\mathrm{H}]^{+}$ & $\mathrm{MW}_{\text {meas }}[\mathrm{M}+\mathrm{H}]^{+}$ \\
\hline CF-KAAYSL-NH ${ }_{2}$ & 21.25 & 1007.8 & 1008.4 \\
\hline CF-KSCYSL-NH ${ }_{2}$ & 21.27 & 1056.7 & 1056.4 \\
\hline CF-GYYNPT-NH ${ }_{2}$ & 21.47 & 1070.8 & 1070.4 \\
\hline CF-KAAYSLGYYNPT & 21.37 & 1704.5 & 1704.4 \\
\hline CF-KSCYSLGYYNPT & 21.28 & 1751.9 & 1752.0 \\
\hline CF-TAKLYPGYANYS-NH ${ }_{2}$ & 21.10 & 1704.5 & 1704.0 \\
\hline CF-YSLGYYNPT-NH 2 & 22.62 & 1433.8 & 1433.7 \\
\hline
\end{tabular}

HPLC: Knauer analytical RP-HPLC (Bad Homburg, Germany); Nucleosil 100-5 C18 (250 mm x $4.6 \mathrm{~mm} ; 5 \mu \mathrm{m}$, $100 \AA ̊)$; eluents: $0.1 \%$ TFA/water (A), $0.1 \%$ TFA in water-CH3CN $(20: 80, \mathrm{v} / \mathrm{v})(\mathrm{B})$ linear gradient elution (0 min $2 \%$ - $30 \mathrm{~min} 90 \%$ ); flow rate $1 \mathrm{~mL} / \mathrm{min}$; detection $\lambda=220 \mathrm{~nm}$.

MS: ESI-MS (Bruker Esquire 3000+ ion trap; Bruker Daltonics, Bremen, Germany); acquisition in 50-2000 m/z range, $4 \mu \mathrm{L} / \mathrm{min} 0.1 \%$ acetic acid in water- $\mathrm{CH} 3 \mathrm{CN}(50: 50, \mathrm{v} / \mathrm{v})$ 
two different sequences to increase the biological activity. In contrast to the CF-labelled hexapeptides, the fluorescent signal intensity of CF-KAAYSLGYYNPT , CF-KSCYSLGYYNPT was the highest following the treatment. This observation was in accordance with the microscopic images and fluorescent signal mainly was detected on the cell surface, and only a small amount of signal concentrated on the cytoplasm (Figure 1C). The cell surface localization was also confirmed by trypsinization that resulted in significantly lower fluorescence intensity (Figure 1B).

To identify receptor specificity, a scrambled variant of KAAYSLGYYNPT (CF-TAKLYPGYANYS-NH ${ }_{2}$ ) was also prepared and no cellular uptake was detected. In addition, the usage of an $\mathrm{N}$-terminally shortened homing peptide (CF-YSLGYYNPT - $\mathrm{NH}_{2}$ ) did not result in binding. These observations suggest that the KCCYSL sequence based peptides provide the receptor binding capacity, but the GYYNPT sequence that does not recognize the HER2 receptor alone, can increase the binding affinity in the combined version.
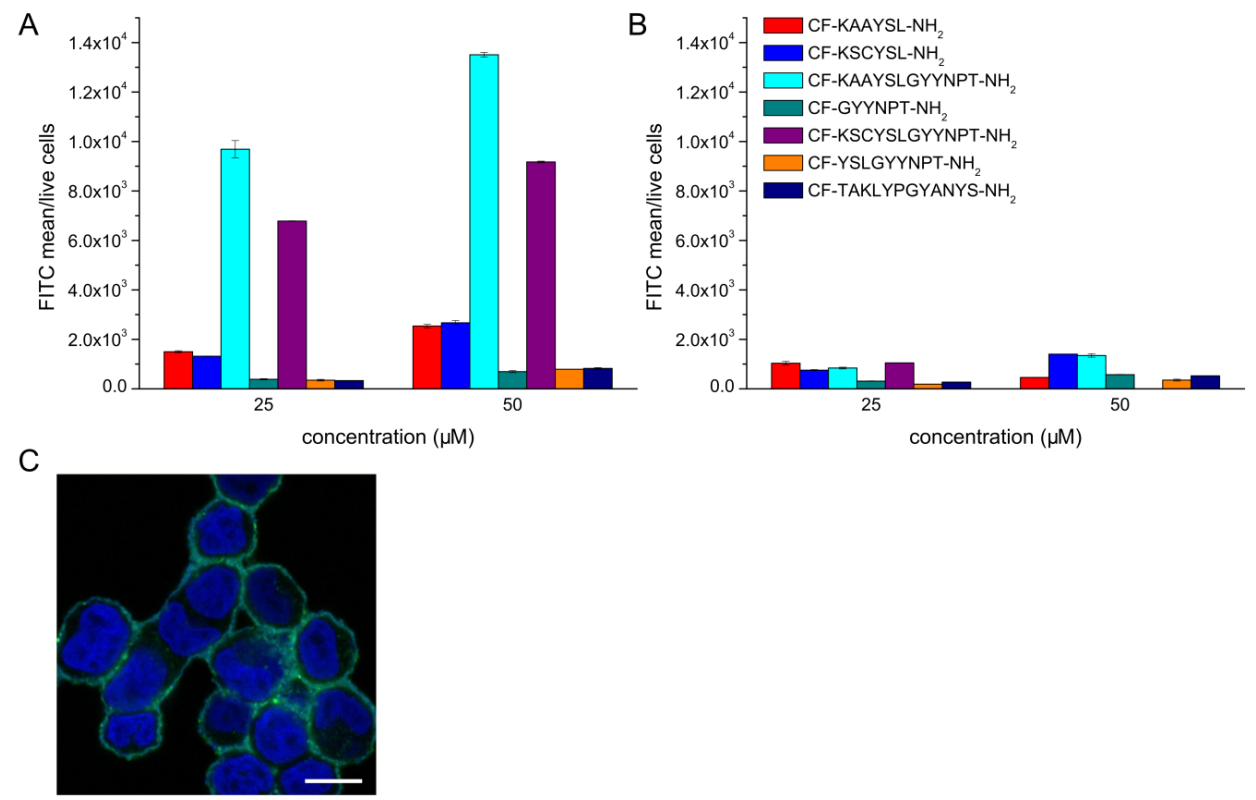

Figure 1: Cellular uptake of CF-labelled peptides by MDA-MB-453 HER2+ breast cancer cells. Uptake was measured after $3 \mathrm{~h}$ treatment (A) without trypsinization and (B) followed by 5 min trypsinization by flow cytometry. (C) Cellular uptake of peptide CF-KAAYSLGYYNPT-NH $\mathrm{H}_{2}$ (green) by MDA-MB-453 cells $(1 \mathrm{~h}$ incubation, concentration: $25 \mu \mathrm{M}$ ) was detected by confocal microscopy. Nuclei are stained with DAPI (blue). The scale bar represents $10 \mu \mathrm{m}$.

PEP-FOLD3.5 prediction method was applied for the study of structure - activity relationship [6,7]. The data indicate that the combined peptides might have helical structure, while the shortened and scrambled ones are disordered (Figure 2). This observation is in accordance with the structural features of an engineered HER2 affibody containing three helical parts [8]. To confirm this structure, further CD measurements are in progress.

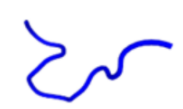

YSLGYYNPT

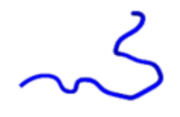

TAKLYPGYANYS

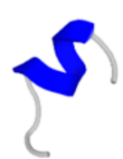

KSCYSLGYYNPT

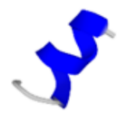

KAAYSLGYYNPT

Figure 2: Predicted structure of peptides by PEP-FOLD3.5 prediction method.

Altogether, the results suggest that the combined peptide is suitable for high affinity receptor binding, but the internalization of the receptor conjugate complex is low. Therefore, these homing peptides may be applied 
for tumor diagnostic (e.g. PET) or selective delivery of radiotracers with therapeutic activity. In addition, the application of extracellular enzyme (elastase, MMPs) cleavable spacers between the homing peptide and an antitumor agent might be a good choice for the development of conjugates as drug delivery systems.

This project was founded by the National Research, Development and Innovation Office (NKFIH K119552, NVKP_16-1-2016-0036), MedInProt, Hungary and Ministry of Human Capacities (1783-3/2018/FEKUTSRA T).

\section{References}

[1] Ménard S.,et al.;Oncogene 22: 6570-6578 (2003) doi: 10.1038/sj.onc.1206779

[2] Lewis Philips GD., et al.; Cancer Res. 68: 9280-9290 (2008) doi: 10.1158/0008-5472.CAN-08-1776

[3] Altmann K-H.; Chimica 72: 154-155 (2018) doi:10.2533/chimia.2018.154

[4] Karasseva NG., et al.; J. Prot. Chem. 21: 287-296 (2002) doi.org/10.1023/A:101974950

[5] Geng L., et al.; Theranostics 6: 1261-1273 (2016) doi: 10.7150/thno.14302

[6] Alland C., et al.; Nucleic Acid Res 33(Web Server issue): W44-49 (2005) DOI: 10.1093/nar/gki477

[7] Néron B., et al.; Bioinformatics 25: 3005-3011 (2009) doi: 10.1093/bioinformatics/btp493

[8] Eigenbrot C., et al.; Proc. Nat. Acad. Sci. USA 107: 15039-15044 (2010) doi: 10.1073/pnas.1005025107 